\title{
Entrepreneurs transnationaux et commerce d'exportation de véhicules d'occasion : la filière Bruxelles-Cotonou
}

Transnational Entrepreneurs and Second-hand Cars Business between Brussels and Cotonou

Empresarios transnacionales y comercio de exportación de vehículos usados: la sucursal Bruselas-Cotonou

\section{Martin Rosenfeld}

\section{(2) OpenEdition \\ Journals}

Édition électronique

URL : https://journals.openedition.org/remi/6399

DOI : 10.4000/remi.6399

ISSN : $1777-5418$

Éditeur

Université de Poitiers

Édition imprimée

Date de publication : 1 juin 2013

Pagination : $57-76$

ISBN : 979-10-90426-08-5

ISSN : 0765-0752

Référence électronique

Martin Rosenfeld, «Entrepreneurs transnationaux et commerce d'exportation de véhicules

d'occasion : la filière Bruxelles-Cotonou », Revue européenne des migrations internationales [En ligne], vol. 29 - n² | 2013, mis en ligne le 01 juin 2016, consulté le 14 avril 2022. URL : http://

journals.openedition.org/remi/6399; DOI : https://doi.org/10.4000/remi.6399 


\section{Entrepreneurs transnationaux et commerce d'exportation de véhicules d'occasion : la filière Bruxelles-Cotonou}

\section{Martin Rosenfeld ${ }^{1}$}

\section{Introduction}

Depuis une dizaine d'années déjà, plusieurs auteurs font état de l'émergence de nouvelles migrations en Belgique (Bribosia et Rea, 2002 ; Martiniello et al., 2010). Ces approches, qui partent du constat de l'insuffisance du modèle d'immigration-intégration pour décrire un certain nombre de nouvelles pratiques migratoires, sont l'enjeu de nombreux questionnements théoriques et méthodologiques.

Dès les textes fondateurs de la sociologie des migrations, l'étranger, l'immigrant est associé à l'image du commerçant : " Toute l'histoire économique montre que l'étranger fait partout son apparition comme commerçant, et le commerçant comme étranger "(Simmel, 2004 [1908] : 54). Alors que cette figure de la migration a été largement étudiée dans le monde anglo-saxon, notamment via les études sur l'ethnic entrepreneurship, elle demeure relativement marginale dans les travaux francophones. Une exception notoire se rapporte aux recherches sur les réseaux commerciaux établis entre les deux rives du bassin méditerranéen (voir par exemple :Tarrius, 1992 ; Missaoui, 1995 ; Manry, 2005 ; Peraldi, 2007 ; Schmoll, 2011). Alors même que de tels réseaux commerciaux prennent place à Bruxelles, ils demeurent encore largement méconnus. Cet article vise donc à mieux comprendre les nouvelles pratiques migratoires en Belgique en s'intéressant plus précisément à la figure de l'entrepreneur migrant. II s'appuie sur une recherche menée sur le commerce d'exportation de véhicules d'occasion entre Bruxelles et Cotonou.

L'étude d'une filière commerciale organisée sur plusieurs places marchandes à travers les continents demande l'usage d'outils méthodologiques spécifiques. Le recours au concept de carrière a ainsi été mobilisé, que ce soit les carrières des entrepreneurs migrants ou bien les " auto-biographies " que lgor Kopytoff

\footnotetext{
1 Assistant-enseignant à I'Université Libre de Bruxelles. Doctorant à l'École des Hautes Études en Sciences Sociales rattaché au CADIS. Chercheur au GERME, Institut de Sociologie, CP 124, Av. Jeanne 44, 1050 Bruxelles ; martin.rosenfeld@ulb.ac.be
} 
(1986) appelait de ses vœux. Suivre le circuit des véhicules d'occasion a aussi été indispensable pour parvenir à rencontrer les personnes impliquées dans ce commerce. C'est donc en menant des enquêtes à Bruxelles entre 2008 et 2011 et lors de trois séjours de plusieurs mois à Cotonou, qu'a pu émerger une compréhension plus générale de la filière commerciale et de ses acteurs. Alors que le contact initial à Bruxelles a été extrêmement réservé, le fait d'avoir pu rencontrer des importateurs à Cotonou dans leur environnement a permis de lever les premiers doutes. Nous avons pu alors travailler de façon régulière avec une cinquantaine d'importateurs et accompagner quelques-uns d'entre eux dans leurs circulations commerciales.

Si notre objectif est bien de contribuer à l'analyse des nouvelles formes de migrations en Belgique, la mobilisation du concept d'entrepreneur migrant semble particulièrement utile pour y parvenir. Dans la première partie de l'article, nous rendons compte des usages de ce concept dans les sciences sociales, ce qui nous permet de questionner le profil migratoire de nos migrants. La seconde partie traite du commerce d'exportation de véhicules d'occasion entre Bruxelles et Cotonou. Elle vient illustrer le type de filière commerciale mise en œuvre par ces entrepreneurs migrants en décrivant comment celle-ci s'insère dans l'axe Bruxelles-Cotonou. Dans les parties suivantes, nous analysons la circulation migratoire et les spécificités du profil des importateurs, ce qui nous permet de nuancer l'approche des migrations sub-sahariennes en Belgique. Enfin, dans la conclusion nous revenons sur les enseignements issus de l'étude du commerce d'exportation de véhicules d'occasion tel qu'il prend place sur l'axe BruxellesCotonou.

\section{La figure de l'entrepreneur migrant dans la littérature}

La question de l'entreprenariat occupe une place importante dans les sciences sociales, que ce soit en anthropologie, en sociologie ou dans les sciences économiques. La partie de la littérature directement en lien avec notre objet d'étude peut être organisée en trois branches principales ; les études africanistes, la littérature - essentiellement anglo-saxonne - sur l'entreprenariat ethnique et les études qui relèvent des sciences économiques. Un rappel des principales orientations de ces trois courants nous permettra de situer notre approche au regard des questionnements de ce champ d'étude.

\section{Approche anthropologique de l'entreprenariat africain}

De nombreux anthropologues africanistes se sont penchés sur la question de l'entreprenariat. II s'agit d'une littérature riche, abondante et bien ancrée ethnographiquement. Les études sur l'entreprenariat africain ont connu un premier développement dans les années 1950-1960. Celles-ci accompagnaient les mouvements d'indépendances et insistaient essentiellement sur les lacunes de ces formes économiques. De nombreux auteurs ont alors produit différentes analyses sur les obstacles aux développements de l'entreprenariat en Afrique. Yves-André Fauré (1994) par exemple propose de distinguer trois domaines particuliers. 
Les obstacles politiques concernent essentiellement la volonté de limiter l'entreprenariat par la classe politique au pouvoir. Le secteur privé peut en effet être perçu comme un obstacle à la logique patrimoniale et clientéliste de certaines élites politiques. Janet Mac Gaffey (1987) suggère, dans le cas de la République Démocratique du Congo, que l'éloignement de Kinshasa a permis l'émergence d'une classe entrepreneuriale à Kisangani et ouvert des opportunités économiques qui sont venues combler l'absence d'investissements du pouvoir central.

En ce qui concerne les obstacles de type économique Yves-André Fauré écrit : "les secteurs agricoles sont dominants, la productivité est faible, les équipements et infrastructures sont inexistants ou défaillants, le revenu national est bas, l'auto-consommation est forte et le secteur des biens interchangeables est réduit, l'épargne nationale est très modeste et l'investissement peu élevé ou ses occasions rares, les marchés intérieurs sont très étroits, les placements se détournent de l'industrie - où les coûts d'investissements sont élevés et la rentabilité du capital relativement faible et à long terme - pour s'orienter dans des secteurs immédiatement plus profitables et/ou plus spéculatifs (immobilier et foncier, commerce intérieur, commerce transfrontalier, activités d'importexport, transports) "(1994: 11-12). Et bien que certains segments des économies africaines aient depuis connus un développement spectaculaire, bon nombre de ces contraintes restent d'actualité dans une grande partie de l'Afrique.

Enfin, les contraintes socio-culturelles insistent sur les difficultés de développement du domaine économique de par son enchâssement dans d'autres sphères de la vie sociale. Depuis les travaux de Karl Polanyi (1983 [1944]), cette interdépendance entre l'économique et l'ensemble des autres sphères d'activités et de relations sociales est bien établie. Cela peut poser certains problèmes supplémentaires dans le contexte africain. Par exemple, dans la difficulté d'accumulation de capital face aux systèmes familiaux de redistribution des richesses. Ou encore dans une vision sorcellaire de la société dans laquelle la réussite économique est perçue comme étant le résultat de pratiques magico-religieuses (Geschiere, 1996).

Ce champ de recherche connaît un nouveau développement dans les années 1990. II semble y avoir alors une volonté de prendre le contre-pied de l'approche pessimiste des années 1960. À partir du constat de la prévalence de la dynamique entrepreneuriale, les travaux se portent sur les causes et les conditions du succès d'entrepreneurs africains. La publication de deux ouvrages collectifs proposant un tour d'horizon de ces études (Gregoire et Labazée, 1994 ; Ellis et Fauré, 1995) témoigne de la fécondité de cette approche.

Au final, alors que l'État est longtemps resté au centre des recherches sur la dimension économique en Afrique (Ellis et Fauré, 1995 : 8), cette littérature a permis de dégager la force entrepreneuriale de certains groupes tels que les Bamilekes (Warnier, 1993), les Haoussas (Grégoire, 1986), les Nandes (MacGaffey, 1987) ou encore les Mourides (Riccio, 2001 ; Bava, 2003) ${ }^{2}$.

2 On notera la volonté de rapprochement entre les travaux français et anglo-saxons, notamment avec la série "Nouvelles tendances de la recherche " qui propose la publication d'un même texte en français dans les Cahiers d'études africaines et en anglais dans la revue African Studies Review. 


\section{L'entreprenariat ethnique}

La littérature consacrée à la question de l'entreprenariat ethnique s'est principalement développée dans le monde anglo-saxon. Deux grandes questions de recherches animent le champ. La première s'intéresse à la capacité de certains groupes de migrants à développer, davantage que d'autres, des activités de type entrepreneuriales; la seconde interroge l'efficacité de l'entreprenariat ethnique comme moyen d'assurer une mobilité sociale. Dans cette logique, Min Zhou propose une définition plutôt générale du concept : " Ethnic entrepreneurs are often referred to as simultaneously owners and managers (or operators) of their own businesses, whose group membership is tied to a common cultural heritage or origin and is known to out-group members as having such traits; more importantly, they are intrinsically intertwined in particular social structures in which individual behavior, social relations, and economic transactions are constrained " (2007: 219).

D'autres auteurs vont contribuer à interroger la question de l'entreprenariat ethnique à partir de trois approches : la théorie de l'intermédiaire minoritaire (middleman minority), le concept d'économie ethnique, et celui d'enclave ethnique.

Proposée initialement par Hubert Blalock (1967), la théorie de l'intermédiaire minoritaire décrit des groupes d'immigrés parvenant à se créer des opportunités économiques en mobilisant différentes ressources de type ethnique, telles que des réseaux d'appartenance ou une langue commune. Edna Bonacich, dans un texte qui a fait date, insiste sur le fait que l'opportunité économique découle de la capacité de ces groupes à se placer dans une position d'intermédiaire. "They tend to concentrate in certain occupations, notably trade and commerce, but also other "middleman" lines such as agent, labor contractor, rent collector, money lender and broker. They play the role of middleman between producer and consumer, employer and employee, owner and renter, elite and masses " (Bonacich, 1973 : 583). Cette position d'intermédiaire, et le fait que l'opportunité économique découle de spécificités ethniques, induisent une mentalité de " séjournant " avec un projet économique temporaire et sans volonté d'assimilation. La théorie de l'intermédiaire minoritaire a été décrite comme une théorie du bouc émissaire (Light, 2005) en référence aux élites coloniales promouvant certains groupes ethniques pour remplir le rôle d'intermédiaire marchand auprès des populations locales. C'est notamment le rôle que les Libanais ont occupé dans les colonies françaises d'Afrique de l'Ouest. Le système inégal de répartition des richesses imposé par le pouvoir colonial génère de la frustration chez les populations locales qui, lorsqu'elle se transforme en colère, se retourne contre les intermédiaires marchands plutôt que contre le système en tant que tel. D'une façon plus générale, Ivan Light et Stavros Karageorgis (1994) critiquent plusieurs continuateurs d'Edna Bonacich qui sont tombés dans des travers culturalistes en imputant à des caractéristiques soi-disant intrinsèques le fait que certains groupes ethniques occupent, davantage que d'autre, une position d'entrepreneur. Ce travers culturaliste découle sans doute du fait que, dans I'absolu, l'approche des minorités intermédiaires se focalise davantage sur la dimension de séjour de ces entrepreneurs - ainsi que la compétition intergroupes et l'hostilité qui en découle - que sur les structures sociales dans lesquelles ces entrepreneurs s'insèrent (Zhou, 2007 : 224). 
Dans la continuité des recherches sur les minorités intermédiaires, le concept d'économie ethnique est proposé dans les années 1970 (Light, 1972 ; Bonacich et Modell, 1980). L'important débat qui s'ensuivit, notamment pour définir ce qui rapproche et distingue l'économie ethnique de l'enclave ethnique, conduit Ivan Light et ses collègues à reformuler le concept afin de lui donner une portée plus générale : "An ethnic economy consists of the self-employed, employers, their co-ethnic employees, and their unpaid family workers. Every ethnic or immigrant group has an ethnic economy, but ethnic economies may be large or small. [...] Large and lucrative ethnic economies require strong entrepreneurial capacity. Entrepreneurial capacity means a group's ability to exploit demand conditions. Entrepreneurial capacity depends upon class and ethnic resources" (Light et Karageorgis, 1994 : 663-664). Cependant, si cette reformulation a permis de gagner en clarté, notamment en définissant un important panel de relations économiques pouvant être rassemblées sous le label d'économie ethnique, on peut se demander si le concept n'est pas devenu tellement large qu'il en a perdu une partie de sa capacité analytique.

Quant à la notion d'enclave ethnique, elle désigne un cas particulier du concept plus général d'économie ethnique. Mais pour être en présence d'une enclave ethnique, deux critères supplémentaires doivent être réunis. Le premier est spatial, l'enclave ethnique désignant une localisation précise rassemblant de nombreux commerces identifiés comme appartenant au même groupe ethnique. Le second relève de la co-ethnicité puisque ne sont concernés que les entrepreneurs faisant travailler des personnes appartenant au même groupe ethnique (Portes et Manning, 1986). Les entrepreneurs actifs au sein d'une enclave ethnique mobilisent régulièrement la solidarité non choisie (bounded solidarity) et la confiance contraignante (enforceable trust) pour garantir le bon déroulement de leur activité commerciale (Portes et Zhou, 1992).

Les tendances récentes de la recherche qui viennent alimenter la réflexion sur le concept d'entreprenariat ethnique mettent l'accent sur l'implication simultanée de groupes migrants dans le pays d'accueil et d'origine en promouvant le concept d'entrepreneur transnational. Bien que cette littérature concerne essentiellement des ethnographies décrivant finement les relations entre dynamiques entrepreneuriales et transnationales, plusieurs tentatives de formalisation théorique ont été proposées (voir entre autres Portes et al., 2002 ; Light, 2007 ; Zhou, 2007).

Alors que la grande majorité de la littérature sur l'entreprenariat ethnique est anglo-saxonne, on citera les travaux de Robert Kloosterman, Joanne Van der Leun et Jan Rath (1999) qui ont mis en lumière l'importance du rôle régulateur de l'État dans le développement des entreprises migrantes. En cela, et contrairement aux pays européens qui imposent des règles strictes d'accès au statut d'entrepreneur (obligation de résidence depuis plusieurs années dans le pays, diplômes conditionnant l'accès à certaines professions), les États-Unis ont une position extrêmement libérale. Si cette dimension de régulation politique n'est donc apparue que très marginalement dans les travaux américains, Ivan Light (2005: 661) souligne qu'il s'agit d'un apport majeur à la théorie de l'entreprenariat ethnique. En France, les travaux sur le " commerce étranger " sont quasi inexistants jusqu'aux années 1990 et restent, à la notable exception d'Emmanuel Ma Mung (1994 et 1996), marginaux jusqu'à ce jour (Berbagui, 2005). 
Les recherches conduites sur les pratiques commerciales développées par les entrepreneurs migrants ont constaté qu'elles reposaient sur des logiques ethniques, que ce soit dans la façon de mobiliser de la main-d'œuvre, du capital ou encore dans le fait de se spécialiser dans la vente de produits destinés à sa communauté d'origine. Dès lors en liant structures d'opportunités du pays d'accueil et les caractéristiques de la communauté migrante les modèles théoriques proposés permettent d'analyser de façon dynamique et historiquement située les stratégies des acteurs qui se construisent dans l'interaction de ces deux forces (Waldinger et al., 2006).

\section{Schumpeter et l'entreprenariat dans les sciences économiques}

La question entrepreneuriale occupe une place importante au sein des sciences économiques, pourtant, la figure de l'entrepreneur migrant n'y prend qu'une place marginale (Swedberg, 2010 : 11). Dans les années 1980, l'entreprenariat est perçu comme la seule façon de résorber à grande échelle l'emploi de l'industrie en déclin. Un engouement se manifeste alors au sein des écoles de commerce pour les études entrepreneuriales ${ }^{3}$.

Aujourd'hui encore de nombreux auteurs se revendiquent du travail pionnier de Joseph Schumpeter qui définit l'entrepreneur comme celui capable de créer de la valeur en agençant des ressources d'une façon nouvelle (1935 [1912]). Ce qui caractérise la définition schumpetérienne de l'entreprenariat, c'est donc sa dimension d'innovation. Israel Drori, Benson Honig et Mike Wright (2009) proposent une typologie des principales formes d'entreprenariat étudiées dans le champ économique.

Alors que l'entreprenariat ethnique (voir supra) s'intéresse aux processus d'immigration, d'assimilation et d'adaptation de communautés ethniques, l'entreprenariat international traite de l'émergence de nouveaux marchés internationaux ainsi qu'aux difficultés spécifiques rencontrées par les entrepreneurs dans l'acquisition de ces marchés. L'entrepreneur rapatrié est celui qui, revenant dans son pays d'origine après plusieurs années d'expérience professionnelle ou d'étude à l'étranger, se lance dans une activité économique. Cela pose la question spécifique du rapatriement du capital humain et social dans le pays d'origine. Enfin, les entrepreneurs transnationaux sont ceux qui migrent tout en développant des liens économiques entre leur pays d'origine et le nouveau pays d'installation. Les études sur les entrepreneurs transnationaux s'intéressent donc à la façon dont ceux-ci développent de nouvelles entreprises commerciales tout en mobilisant simultanément les ressources sociales et économiques de plus d'un pays. Bien que chacune de ces orientations de recherches ait produit une littérature importante, un manque de structuration théorique semble les empêcher de s'organiser en un champ d'étude cohérent. Cela se marque notamment par l'absence de consensus autour d'une définition commune du concept d'entrepreneur (Howorth, Tempest et Coupland, 2005).

3 Le champ se structure également autour de revues spécialisées : Journal of Business Venturing et American Journal of Small Businesses - qui deviendra Entrepreneurship Theory and Practices - pour les Etats-Unis ; Small Business Economics et Entrepreneurship and Regional Development pour I'Europe. 
Une dimension importante de l'approche entrepreneuriale dans les sciences économiques concerne l'opérationnalisation des résultats; alors que les sciences sociales se sont plutôt intéressées au " pourquoi ", aux causes et aux effets de la posture entrepreneuriale, les sciences économiques se sont davantage focalisées sur le " comment " (Jarillo et Stevenson, 1990). En outre on constate avec la formalisation du paradigme entrepreneurial qu'une tension se fait jour au sein des études économiques entre théoriciens et praticiens. Pour William Bygrave par exemple, si les recherches se doivent de garder une dimension empirique et opérationnalisable, il constate qu'elles prennent une dimension de plus en plus théorique, qui mobilisent des techniques statistiques complexes et des bases de données de deuxième main au détriment d'études empiriques et longitudinales (Bygrave, $2007: 20$ ).

Plus directement en lien avec l'objet qui est le nôtre, on retiendra que nombre d'études économiques cherchent à mettre en évidence les avantages comparés d'entrepreneurs étrangers par rapport aux entrepreneurs locaux (pour une revue des travaux, voir Ramachandran et Shah, 1999 : 74), que ce soit par exemple dans l'accès au crédit ou à l'information, ou encore dans la mobilisation de différents types de réseaux (McCabe et al., 2005).

\section{Mobilisation du concept d'entrepreneur migrant dans l'étude d'une filière commerciale transnationale}

Au regard de la littérature sur le concept d'entrepreneur, et alors que de nombreuses définitions ont été proposées, nous privilégions dans notre étude une approche qui relève du mouvement de recherche africaniste : " un entrepreneur est un agent économique qui mobilise durablement travail et capital afin de produire ou d'échanger des biens ou services et d'obtenir un revenu " (Fauré, 1994 : 70). Cette définition très large a le mérite d'englober un nombre conséquent d'acteurs permettant ainsi de mener un travail d'analyse comparatif entre les différents importateurs de véhicules d'occasion que nous avons rencontrés. De plus, elle s'applique à un contexte de recherche proche du nôtre. En effet, l'étude menée parYves-André Fauré sur les entrepreneurs africains se situe en Côte-d'Ivoire et privilégie la dimension ethnographique.

Les différentes orientations théoriques présentées ci-dessus nous ont permis de dégager des questions relatives à la figure de l'entrepreneur migrant. Elles se rapportent à l'organisation de la filière commerciale et au profil des importateurs. La première question à laquelle nous aurons à répondre concerne les types de filières commerciales mises en œuvre par les entrepreneurs migrants et la façon dont ces filières s'installent en Belgique. La seconde question a trait au profil migratoire de ces entrepreneurs migrants. En quoi le profil des importateurs de véhicules permet-il de venir diversifier les figures des migrations de travail en Belgique ? C'est en mobilisant le matériel de terrain issu de la filière Bruxelles-Cotonou d'exportation de véhicules d'occasion que nous tenterons d'apporter des réponses à ces questions. 


\section{Le commerce d'exportation de véhicules d'occasion à Bruxelles}

La filière Bruxelles-Cotonou d'exportation de véhicules d'occasion est une activité commerciale développée par des personnes issues de l'immigration et exclusivement destinée à d'autres migrants. L'activité commerciale se caractérise ainsi par une forte dimension ethnique. Bruxelles, plus particulièrement le quartier Heyvaert situé le long du canal traversant la ville, s'est progressivement constitué en une place marchande qui occupe une position centrale dans cette filière commerciale transnationale. La compréhension du profil des importateurs de véhicules d'occasion nécessite d'abord une description du contexte dans lequel ils opèrent, ce que nous ferons ci-dessous. Cette description sera ensuite complétée par quelques précisions sur les modalités de fonctionnement de I'activité commerciale.

\section{Description du contexte urbain}

Le commerce de véhicules d'occasion se concentre, à Bruxelles, sur quelques rues du quartier Heyvaert, lequel se caractérise par des marqueurs urbains forts. Le plus important est le canal qui dessine une frontière spatiale et symbolique au cœur de la ville. Le canal, qui connecte le port de Bruxelles à la côte, a joué un rôle important dans le développement économique et industriel de la ville. On trouve donc de nombreux bâtiments industriels sur chacune de ses berges. Historiquement, ce quartier ouvrier rassemblait une population au profil socio-économique plus défavorisé que dans le reste de la ville. Si aujourd'hui certains de ces bâtiments industriels ont perdu leur fonction première, le canal reste pourtant, et ce quel que soit l'indicateur mobilisé - niveau d'étude, taux d'emploi, revenu moyen -, une zone de pauvreté qui traverse le centre-ville.

Les abattoirs d'Anderlecht constituent un second marqueur urbain. Situés au début de la rue Heyvaert, ils ont longtemps été le moteur de l'activité économique du quartier. Cependant, le durcissement des règles d'hygiène, suite à l'introduction d'une nouvelle directive européenne dans les années 1990, a rendu difficile le maintien de l'abattage en plein centre-ville. Le site a donc réduit son activité à la découpe du bétail et la majorité des grossistes installés à proximité des abattoirs ont déménagé à la périphérie de la ville.

Le troisième marqueur urbain est la gare du Midi. La plus grande gare du pays, bien qu'elle soit un peu plus éloignée du quartier Heyvaert, n'en joue pas moins un rôle clé dans son développement - ou plutôt son non développement. En effet, le projet des responsables politiques locaux était de transformer en quartiers d'affaires les espaces autour de la gare du Midi, à l'image de ceux qui se sont construits depuis les années 1960 près de la gare du Nord. Le bâti étant voué à la destruction, tout projet de rénovation ou d'investissement a été limité au maximum. Finalement les tours de bureaux ne verront jamais le jour, mais les effets de cette politique se font tout de même sentir : chancres urbains, incohérence dans l'application des règles urbanistiques, passage anarchique d'une zone principalement industrielle vers une zone d'habitation et, enfin, délabrement généralisé de l'état du bâti. La transformation de la morphologie de l'espace a fait du quartier Heyvaert une zone de relégation pour différentes vagues migratoires venues s'y installer. Ce quartier est en effet l'une des portes 
d'entrée pour les primo-arrivants à Bruxelles qui trouvent à se loger à faible coût. Mais cet espace a aussi vu le commerce de véhicules d'occasion progressivement s'y implanter.

\section{Quartier Heyvaert : de la boucherie aux véhicules d'occasion}

Le développement du commerce de véhicules d'occasion dans le quartier Heyvaert est le résultat de multiples facteurs, mais plusieurs événements clés ont joué un rôle suffisamment prépondérant dans ce processus pour devoir être cités. Deux personnages en particulier semblent avoir largement contribué à faire de ce quartier ce qu'il est aujourd'hui. Le premier, monsieur A., est un consignataire libanais. Venu en Belgique grâce à une bourse d'études en médecine, il se lance rapidement dans les affaires qui vont connaître un développement important à la faveur de la guerre civile au Liban. En effet, I'insécurité des transports publics au Liban s'est accompagnée d'une demande croissante de voitures bon marché. Dès lors, l'exportation de véhicules d'occasion d'Europe vers le Liban est florissante jusqu'à la fin de la guerre civile. monsieur A. va ensuite chercher un marché de substitution pour ses véhicules d'occasion. À cette époque, la demande africaine est en plein essor, monsieur A. prend alors deux décisions importantes : transférer son activité du Moyen-Orient vers l'Afrique sub-saharienne et déplacer le centre névralgique du transport de véhicules de la Méditerranée vers l'océan Atlantique. Pour ce faire, un accord est conclu avec l'un des plus grands armateurs italiens qui desservira les principaux ports africains à partir d'Anvers. Les facilités portuaires, la rapidité de la traversée et des tarifs attractifs contribuent au développement du port d'Anvers jusqu'à en faire le premier port européen d'exportation de véhicules d'occasion, loin devant les ports méditerranéens, mais aussi en avance sur Rotterdam ou Hambourg.

Si le marché belge de l'occasion présente un attrait pour les clients africains, c'est que l'enjeu a consisté à leur proposer une solution globale pour la prise en charge de leur véhicule. C'est ce service qu'offre monsieur $A$. en proposant à ses clients qui achètent un véhicule de le stocker dans sa société avant de I'acheminer jusqu'au port d'Anvers puis de l'envoyer vers l'Afrique. Dès lors que ce service est proposé dans le quartier Heyvaert, d'autres garagistes spécialisés dans l'exportation de véhicules d'occasion vont chercher à s'y installer. L'augmentation de garages spécialisés dans la vente de véhicules d'occasion va à son tour attirer d'autres sociétés concurrentes de consignation.

Un second personnage joue un rôle clé. madame F. vit et travaille depuis longtemps dans le quartier Heyvaert. Son mari dirige une société de vente de viande en gros et lorsque cette activité commence à décliner, madame F. décide de se diversifier en organisant chaque semaine une vente de gré à gré de véhicules d'occasion. Elle se trouve donc à l'intersection entre, d'une part, les garagistes à la recherche de grands espaces d'exposition pour leurs véhicules d'occasion et, d'autre part, les grossistes en viande cherchant de plus en plus à quitter le quartier au profit d'installations neuves et conformes aux nouvelles directives européennes. Cette position lui permettra de jouer un rôle d'intermédiaire en connectant les protagonistes de ces deux secteurs d'activités. Ce faisant, le quartier Heyvaert autrefois tout entier tourné vers la boucherie va de plus en plus organiser son activité autour de l'exportation de véhicules d'occasion. 


\section{Modalités du fonctionnement de la filière commerciale}

Les pionniers du commerce d'exportation de véhicules d'occasion à Bruxelles sont les Libanais qui, les premiers, ont offert une solution globale aux clients cherchant non seulement à acheter un véhicule, mais également à organiser son transfert vers l'un des principaux ports africains. Aujourd'hui encore ce sont des membres de la communauté libanaise qui, à Bruxelles, occupent les postes clés de cette activité commerciale. Propriétaires des plus grands garages et, surtout, des principales sociétés de consignation, ils occupent une position d'intermédiaire avec les armateurs de navire et prennent en charge le transfert des véhicules vers l'Afrique. Toute cette infrastructure est destinée à leurs clients, importateurs de véhicules d'occasion qui viennent prioritairement d'Afrique sub-saharienne ${ }^{4}$. On rencontre à Bruxelles des importateurs de nationalités diverses et les destinations possibles pour les voitures sont donc nombreuses: Douala, Abidjan, Pointe-Noire, Lomé, etc. ; parmi celles-ci, la destination la plus courue est Cotonou, au Bénin. Cela est dû au fait que le Bénin occupe une position " d'État entrepôt " (Igué et Soulé, 1992) spécialisé dans l'importation et la réexportation de marchandises à l'échelle de l'Afrique de l'Ouest. Cette situation de plaque tournante dans la redistribution des véhicules en Afrique est assez comparable à celle de place marchande que Bruxelles occupe dans le rassemblement et la concentration des véhicules en Europe. Parmi les multiples branches possibles de cette activité commerciale, nous avons donc choisi de nous concentrer sur l'axe Bruxelles-Cotonou.

Les véhicules d'occasion à destination de Cotonou passent par deux types de canaux. Le premier vient d'être brièvement évoqué. Il s'agit d'importateurs, essentiellement béninois, qui se rendent en Belgique pour acheter un lot de véhicules qu'il confie ensuite à un consignataire qui prend en charge son transfert jusqu'au port d'Anvers puis le convoyage par bateau. Trois semaines plus tard, l'importateur réceptionne ses véhicules à Cotonou afin de les mettre en vente sur l'espace qu'il occupe dans un des parcs de vente de véhicules d'occasion. Le second canal procède de la même façon, excepté qu'il se passe de la présence des importateurs à Bruxelles. Dans ce cas, les consignataires libanais prennent en charge la réception puis l'acheminement de véhicules d'occasion pour des importateurs libanais restés à Cotonou. Cette sous-filière ethnique ${ }^{5}$ libanaise offre aux importateurs libanais qui travaillent à Cotonou d'importantes facilités en termes d'acheminement, de paiement ou de choix des véhicules.

À l'opposé de toutes dérives essentialistes, ce qui fait sens ici est la façon dont le groupe se constitue et se définit. Dans le cadre de la filière BruxellesCotonou, ces catégories ethniques semblent se superposer à des catégories d'appartenance nationales ; Libanais d'une part, Béninois de l'autre. Si ces catégories d'appartenance parviennent à gommer une série de réalités sociales plus fines - être Fon ou Yoruba pour les importateurs béninois, appartenir à différentes confessions religieuses pour les Libanais - c'est parce qu'elles revêtent un sens économique pour les acteurs. En effet, désigner d'autres acteurs de

4 Pour une description plus précise de ces différentes fonctions, se référer à la typologie des acteurs du commerce d'exportation de véhicules d'occasion (Rosenfeld, 2009).

5 Ethnicité est entendu ici au sens de Jocelyne Streiff-Fénart (1997). 
l'activité commerciale comme des membres du groupe permet d'en faire des partenaires potentiels. Cette potentialité s'est traduite, dans la situation décrite, par la création d'une sous-filière d'exportation de véhicules d'occasion dont I'ensemble des intermédiaires sont désignés comme Libanais.

Il existe donc deux voies d'approvisionnement pour les véhicules d'occasion correspondant à deux profils bien distincts d'importateurs. Ces profils s'organisent sur la base d'un critère d'appartenance ethnico-national ; les importateurs libanais d'une part et béninois de l'autre. Aucun importateur libanais ne se déplace lui-même pour acheter ses véhicules d'occasion. À l'inverse, très peu d'importateurs béninois parviennent à se faire envoyer leurs véhicules directement à Cotonou, ils sont donc obligés d'aller les chercher sur place.

\section{Pratique migratoire des entrepreneurs dans l'importation de véhicules d'occasion}

Alors qu'ils occupent la même fonction - importateur de véhicules d'occasion - et qu'ils travaillent au sein de la même filière commerciale, l'axe Bruxelles-Cotonou, les importateurs libanais et béninois ont des pratiques migratoires radicalement différentes. La compréhension de leur statut d'entrepreneur migrant passe donc par une description plus fine de ces pratiques.

\section{Entrepreneurs transfrontaliers béninois et territoire circulatoire}

Les importateurs béninois sont amenés à faire des allers-retours réguliers entre Cotonou et Bruxelles afin d'acheter eux-mêmes leurs véhicules d'occasion. Le principe est le suivant ; un capital est rassemblé afin que l'importateur parte à Bruxelles acheter un lot de véhicules. Ceux-ci sont expédiés à Cotonou puis vendus sur place. Dès que la vente des véhicules a permis de reconstituer le capital, un nouveau séjour est organisé à Bruxelles afin d'acheter un autre lot de véhicules. De cette façon, les importateurs béninois peuvent effectuer jusqu'à trois ou quatre allers-retours par an avec chaque fois un budget d'une quinzaine de milliers d'euros, ce qui équivaut à l'achat d'une douzaine de véhicules.

Ces nombreux allers-retours peuvent correspondre à plusieurs mois par an de présence à Bruxelles. II en est ainsi pour les importateurs béninois, mais également pour les importateurs d'autres nationalités africaines actives dans cette filière commerciale. Nous avons donc, directement lié à la filière BruxellesCotonou d'exportation de véhicules d'occasion, une importante présence africaine dans le quartier Heyvaert. Celle-ci a progressivement amené le développement de services annexes ; restaurants africains, épicerie vendant des produits exotiques, centre d'appels téléphoniques internationaux bon marché ou encore plusieurs églises pentecôtistes d'obédience africaine.

En interrogeant les importateurs béninois sur leurs séjours à Bruxelles, il est étonnant d'observer à quel point ils sont concentrés sur leur activité commerciale. Tout ce qui n'est pas directement lié à l'achat de véhicule semble être considéré comme une perte de temps et d'argent. Ainsi, malgré plusieurs mois de présence annuelle pour certains d'entre eux, seul le quartier Heyvaert est fréquenté. Ils logent dans l'un des hôtels bon marché des environs et l'ensemble des acteurs de l'activité est rassemblé sur cette place marchande où différents 
services annexes liés au confort du séjour se sont développés. Les autres quartiers de la ville ne sont ni connus ni fréquentés. Nous sommes ainsi dans un profil migratoire complètement différent de celui du transmigrant.

Malgré une coprésence régulière à Bruxelles et à Cotonou, I'on ne retrouve pas chez les importateurs béninois les multiples formes de liens - familiaux, économiques, sociaux, organisationnels, religieux ou encore politiques - décrits dans la définition initiale du concept de transmigrant (Basch et al., 1994). II faut dire que, contrairement à la plupart des groupes migrants étudiés par les anthropologues qui ont proposé ce concept, les importateurs béninois de véhicules $d^{\prime}$ occasion ne sont pas venus en Belgique vendre leur main-d'œuvre - ce qui demande un certain degré d'implication dans la société où l'on vit - mais des marchandises. Cette distinction, clairement mise en évidence par Michel Bruneau (2004), semble ici opérationnelle et conduit, pour caractériser ces importateurs béninois, à mobiliser le concept d'entrepreneurs transfrontaliers, actifs au sein d'un territoire circulatoire dans les travaux d'Alain Tarrius (1993 et 2001).

La notion de territoire circulatoire développée par Alain Tarrius reprend une intuition proposée par Maurice Halbwachs dans La topographie légendaire des Évangiles en Terre Sainte et discutée également par Michel Foucault avec son concept d'hétérotopie (2004 [1967]), à savoir la possibilité de superposition de plusieurs territoires sur un même espace. Là où c'est la mémoire collective qui, chez Maurice Halbwachs, conduit différents groupes à développer des territoires mythiques se superposant sur un même espace - et s'articulant souvent autour des mêmes lieux -, c'est la mobilité qui occupe cette fonction chez Alain Tarrius : "Ces territoires, nous les nommons territoires circulatoires. Cette notion constate la socialisation d'espaces supports à des pratiques de mobilité. Elle introduit une double rupture dans les acceptions communes du territoire et de la circulation; en premier lieu elle nous suggère que l'ordre né des sédentarités n'est pas essentiel à la manifestation du territoire, ensuite elle exige une rupture avec les conceptions logistiques des circulations, des flux, pour investir de sens social le mouvement spatial " (2001: 45).

Ce sont donc les multiples liens sociaux développés par le circulant le long de son trajet migratoire qui permettent d'associer différents espaces au sein d'un même territoire circulatoire. Dans le cas des réseaux de commerces souterrains étudiés par Alain Tarrius de part et d'autre de la Méditerranée, le territoire circulatoire désigne donc l'ensemble des espaces - lieux de passages, de frontières, d'étapes - investis socialement par l'entrepreneur transfrontalier afin de rendre possible son activité commerciale. Si cet entrepreneur est défini comme transfrontalier, c'est parce que l'intérêt économique de son activité découle de sa capacité à traverser les frontières. Ces frontières peuvent être à la fois sociales - l'opportunité économique provient alors de la connexion de groupes n'entretenant que peu de relations entre eux - ou bien physiques, et c'est bien souvent la capacité à circonvenir le système de taxation douanière qui permet de créer de la richesse (Peraldi, 2007).

\section{La diaspora commerciale libanaise}

Contrairement aux importateurs béninois, les Libanais ne se déplacent pas pour acheter les véhicules d'occasion. Ce sont leurs intermédiaires libanais qui 
se chargent, à Bruxelles, de toutes les opérations nécessaires à l'expédition de la marchandise. Cependant, ce type d'organisation commerciale comporte des risques : pas de contrôle sur l'état des véhicules achetés, pas de garantie d'envoi du véhicule, factures impayées, etc. Les importateurs libanais parviennent à minimiser ces risques en travaillant au sein d'une "diaspora commerciale" au sens décrit par Abner Cohen (1971) : un groupe qui met à profit une même appartenance ethnique pour résoudre des problèmes techniques liés à une activité commerciale commune. En outre, l'implantation à Bruxelles d'une communauté libanaise qui a acquis de l'expérience dans le commerce est une garantie de sérieux des transactions. Alors que les pionniers de l'activité appartiennent essentiellement à une élite chrétienne maronite venue en Belgique dans le cadre d'études supérieures, une deuxième vague arrive dans les années 1990 et s'implante à son tour dans le quartier Heyvaert. Aujourd'hui ce sont les enfants des pionniers, pour la plupart nés en Belgique, qui ont repris la gestion des grands garages et des sociétés de consignation.

Pour ces entrepreneurs, la confiance joue un rôle majeur dans les échanges commerciaux en ce qu'elle permet la création de réseaux d'approvisionnement. C'est la capacité à développer ce type de réseaux d'approvisionnement qui distingue les importateurs qui travaillent à distance de ceux qui viennent s'approvisionner sur place (Rosenfeld, 2012).

Il convient donc de revenir au questionnement de départ de ce texte afin d'évaluer l'impact que ce type d'activité commerciale - et les différentes formes de circulation qu'elle induit - peut jouer dans les nouvelles pratiques migratoires observées en Belgique.

\section{Quand l'Afrique investit en Europe}

La recherche menée sur la place marchande installée dans le quartier Heyvaert nous a permis d'éclairer différentes pratiques liées au commerce d'exportation de véhicules d'occasion. Ce faisant, l'intérêt porté à la figure de l'entrepreneur migrant nous amène à revoir une série de propositions sur les migrations prenant place en Belgique.

Tout d'abord, le sens des flux financiers mérite d'être souligné. Des capitaux africains sont investis en Europe afin d'acquérir des biens " en fin de vie " qui seront consommés dans les pays du Sud. Les raisons qui permettent d'expliquer l'intérêt financier d'un tel circuit économique sont évidemment multiples : absence de chaînes de production de véhicules dans une large partie du continent africain, niveau de vie qui rend prohibitif I'achat d'un véhicule neuf, mais surtout l'écart entre l'Europe et de nombreux pays africains concernant les coûts de réparation et les normes imposées par le contrôle technique. Un véhicule nécessitant un investissement conséquent pour correspondre aux normes techniques imposées en Europe pourra être mis en circulation et entretenu à bon compte en Afrique, d'où l'intérêt pour ce type de marchandises.

Le fait que nous soyons ici en présence d'entrepreneurs sub-sahariens dans ce cas béninois - qui investissent des sommes conséquentes en Belgique permet d'aller à l'encontre de nombre de stéréotypes concernant les migrants sub-sahariens, dont le plus répandu est celui " de venir prendre d'assaut le 
système social de l'Europe forteresse ". Comme l'explique monsieur O., importateurs béninois de véhicules d'occasion :

" (...) Ce sont des gens qui sont bien établis ici, qui n'ont rien à chercher en Europe.

Et l'inquiétude de l'Occident, c'est d'octroyer des visas aux gens qui vont rester et ne plus revenir et leur greffer des problèmes là-bas. Moi, maintenant, qu'est-ce qu'on veut m'offrir en Europe qui me ferait m'installer là-bas? Je ne vois pas ! "(Cotonou,

22/06/2010).

S'installer en Europe non seulement compliquerait le déroulement de l'activité commerciale, mais de plus le niveau de vie de ces entrepreneurs est bien supérieur au Bénin. Ce qui n'empêche pas que les importateurs de véhicules d'occasion restent soumis à un régime drastique d'octroi de visa, ce qui est pour eux autant une contrainte qu'une source de frustration. Les États-Unis ne s'y sont pas trompés et leur système d'octroi de business visa leur permet aujourd'hui de capter un tiers du marché béninois d'importation de véhicules d'occasion là où, il y a seulement dix ans, il était encore complètement absent (CNCB, 2009).

Une seconde dimension a trait au type de mobilité mis en œuvre par les acteurs de cette activité commerciale. À côté des Libanais déjà bien installés en Belgique, nous avons vu que des importateurs sub-sahariens font régulièrement des allers-retours afin de s'approvisionner en véhicules. Bien loin du schéma classique d'immigration-intégration, ces commerçants ont des pratiques de circulation migratoire (Doraï et al., 1998) et leur implication sur les lieux de passages, comme à Bruxelles, reste exclusivement centrée sur leur activité commerciale. Si c'est bien de leur mobilité qu'ils tirent leurs principales ressources économiques, c'est au pays d'origine qu'ils vivent leur quotidien.

Enfin, une troisième dimension se rapporte au fait que la filière BruxellesCotonou d'exportation de véhicules d'occasion représente une forme particulière de commerce dans la ville. II s'agit en effet d'une activité commerciale mise en œuvre par des personnes issues de l'immigration et destinée à d'autres migrants. L'activité s'est développée à l'initiative de personnes d'origine libanaise. Quant aux clients, il s'agit d'entrepreneurs migrants. L'activité commerciale se caractérise donc par une forte dimension ethnique. II s'agit également d'une opportunité économique qui, de par sa spécificité, échappe en grande partie aux acteurs belges. Une exception est celle de madame F, doyenne parmi les vendeurs de véhicules d'occasion du quartier Heyvaert, qui est belge d'origine et femme. Celle-ci a joué un rôle clé d'intermédiaire au moment de la transition économique de ce quartier autrefois dédié à la boucherie. Mais il faut souligner que si les Belges impliqués dans la filière Bruxelles-Cotonou d'exportation de véhicules d'occasion sont rares, ce n'est pas pour autant que cette activité ne bénéficie pas indirectement à un nombre important d'entre eux ; le port d'Anvers assure le transport de plusieurs dizaines de milliers de véhicules d'occasion par an.

\section{Conclusion}

Dans un premier temps, et en nous appuyant sur différents travaux issus de la littérature sur les entrepreneurs migrants, nous avons décrit le fonctionnement d'une filière d'exportation de véhicules d'occasion et montré que si les 
voitures d'occasion traversent les continents, il en va de même des entrepreneurs migrants qui organisent ce commerce entre Bruxelles et Cotonou.

L'intérêt porté à la filière d'exportation de véhicules d'occasion a également révélé l'importance d'une activité commerciale pourtant peu visible à Bruxelles. Chaque mois, près de 10000 véhicules quittent le quartier Heyvaert en direction du port d'Anvers afin d'être expédiés vers I'Afrique de I'Ouest (ECSA, 2010 : 18). Cet aspect nous a permis de mettre en évidence le côté double de la dimension internationale de Bruxelles. Si les responsables politiques mettent systématiquement en avant l'image de Bruxelles capitale de l'Europe et ville globale attirant des migrants hautement qualifiés, il en existe pourtant une autre. Carrefour commercial et place marchande centrale dans une filière économique, nous avons montré que cette autre Bruxelles est tout aussi internationale que la première, mais rarement reconnue et valorisée comme telle.

Dans un second temps, en étudiant le commerce d'exportation de véhicules d'occasion, nous avons pu mettre en évidence une dimension moins connue des migrations d'Afrique sub-saharienne prenant place en Belgique. En centrant la focale sur les entrepreneurs, nous avons décrit un profil relativement spécifique de migrants. Ce faisant, $c^{\prime}$ est également une diversification des profils de migrants sub-sahariens présents en Belgique qui est proposée. Premièrement, il s'agit d'entrepreneurs africains qui viennent investir leur capital en Belgique. Deuxièmement, ces entrepreneurs développent leur activité économique à partir de leur mobilité. Nous sommes donc en présence d'entrepreneurs béninois qui n'ont, a priori, aucun intérêt à chercher à s'installer en Belgique. Troisièmement, ces importateurs béninois maintiennent un ancrage fort dans leur espace national. Malgré une circulation importante et une coprésence entre Bruxelles et Cotonou, leur implication à Bruxelles est exclusivement centrée sur l'activité commerciale. Les autres sphères de la vie sociale n'étant pas investies, ces entrepreneurs n'ont pas un profil de transmigrants (Basch et al. : 1994).

En nous intéressant aux pratiques migratoires des acteurs de la filière, nous avons pu identifier un groupe d'importateurs libanais que nous avons qualifié de diaspora commerciale. Les Libanais combinent, en effet, plusieurs caractéristiques habituellement associées à une diaspora (voir Cohen, 2008 : 17). II s'agit d'un groupe largement dispersé ${ }^{6}$ suite à la guerre civile de 1975-1990, qui maintient son attachement au pays d'origine et qui développe des activités commerciales communes (Bruneau, 2004 ; Cohen, 2008). Dans le cas que nous avons étudié, les importateurs libanais de Cotonou travaillent avec des intermédiaires libanais installés sur la place marchande bruxelloise.

Bien qu'ils soient des importateurs de véhicules actifs dans la même filière commerciale, nous avons montré que les importateurs béninois n'ont pas le même profil migratoire que les importateurs libanais. La faible implication des importateurs béninois à Bruxelles empêche de les définir comme une diaspora ou de mobiliser la littérature sur le transnationalisme pour caractériser leurs pratiques. Ils se présentent plutôt comme des entrepreneurs transfrontaliers au sein d'un territoire circulatoire (Tarrius, $1993 ; 2001)$. En combinant une approche

6 Xavier Auregan (2012) estime ainsi qu'il y a deux fois plus de personnes d'origine libanaise qui vivent à travers le monde qu'au Liban. 
anglo-saxonne qui mobilise les concepts de diaspora et de transnationalisme et une approche francophone formulée initialement autour de l'étude de réseaux commerciaux souterrains prenant place en Méditerranée, nous avons pu montrer, au sein d'une même activité commerciale, que le profil migratoire des entrepreneurs libanais était radicalement différent de celui des béninois.

La dimension transnationale de notre approche a permis de mettre en évidence de nouvelles formes de migrations économiques prenant place dans une période où l'immigration de travail est restreinte. Alors que la plupart des études sur les travailleurs migrants sont focalisées sur les salariés, nous nous sommes intéressés aux entrepreneurs. Ce faisant, nous avons montré que la figure de l'entrepreneur est intéressante au regard de la dimension d'innovation mise en évidence par Schumpeter. C'est en combinant mobilité migratoire et ingéniosité dans le contournement des réglementations douanières que I'entrepreneur migrant parvient à produire des opportunités économiques. Ce constat est d'autant plus important dans une période de crise économique, car il démontre que les entrepreneurs migrants sont capables de passer outre la pression de plus en plus forte à la restriction de l'immigration de travail.

\section{Références bibliographiques}

Auregan Xavier (2012) "Communauté " libanaise en Afrique de l'Ouest, Diploweb, [en ligne] consulté le 28/03/2013. URL : http://www.diploweb.com/ Communaute-libanaise-en-Afrique-de.html

Basch Linda, Glick Schiller Nina and Szanton Blanc Cristina (1994) Nations Unbound:Transnational Projects, Postcolonial Predicaments, and Deterritorialized Nation-States, Amsterdam, Overseas Publishers Association, 344 p.

Bava Sophie (2003) De la "Baraka aux affaires " : ethos économico-religieux et transnationalité chez les migrants sénégalais mourides, Revue Européenne des Migrations Internationales, 19 (2), pp. 69-84.

Berbagui Dalila (2005) Commerce et petite entreprise étrangère dans la ville (1980-2002), Ethnologie française, 35 (1), pp. 109-115.

Blalock Hubert M. Jr (1967) Toward a Theory of Minority Group Relations, New York, John Wiley, $227 \mathrm{p}$.

Bonacich Edna (1973) ATheory of Middleman Minorities, American Sociological Review, 38 (5), pp. 583-594.

Bribosia Emmanuelle et Rea Andrea (Éds.) (2002) Les nouvelles migrations. Un enjeu européen, Bruxelles, Éditions Complexes, 288 p.

Bruneau Michel (2004) Diasporas et espaces transnationaux, Paris, Anthropos, $250 \mathrm{p}$.

Bygrave William (2007) The entrepreneurship paradigm (I) Revisited, in Helle Neergaard and John Ulhøi Eds., Handbook of Qualitative Method Research in Entrepreneurship, Cheltenham, Edward Elgar Publishing Limited, pp. 17-48.

Cohen Abner (1971) Cultural strategies in the organization of trading diasporas, in Claude Meillassoux Ed., The Development of Indigenous Trade and Markets in West Africa, London, Oxford University Press, pp. 266-281. 
Cohen Robin (2008) Global Diasporas. An introduction, Londres and New York, Routledge, $219 \mathrm{p}$.

Conseil National des Chargeurs du Bénin (2009) Bulletin statistique des transports 2008, Cotonou, CNCB.

Doraï Kamel, Hily Marie-Antoinette, Loyer Frantz et Ma Mung Emmanuel (1998) Bilan des travaux sur la circulation migratoire, Poitiers, ministère de la Solidarité et de l'Emploi, Migrinter, $105 \mathrm{p}$.

Drori Israel, Honig Benson andWright Mike (2009)Transnational Entrepreneurship: An Emergent Field of Study, Entrepreneurship Theory and Practice, 33 (5), pp. 1001-1022.

Ellis Stephen et Fauré Yves-André (Éds.) (1995) Entreprises et entrepreneurs africains, Paris, Karthala et ORSTOM, 640 p.

European Center for Strategic Analysis (2010) Prospection du marché et étude de faisabilité pour les trafics ro-ro par voie navigable dans le port de Bruxelles, en mettant l'accent sur les véhicules (neufs ou d'occasion), Bruxelles, 89 p.

Fauré Yves-André (1994) Petits entrepreneurs de Côte d'Ivoire. Des professionnels en mal de développement, Paris, Karthala, 392 p.

Fauré Yves-André (1992) Entrepreneurs et entreprises d'Afrique subsaharienne. Sélection bibliographique, Paris, UREF/AUPELF, 20 p.

Foucault Michel (2004 [1967]) Des espaces autres, Empan, 2 (54), pp. 12-19.

Geschiere Peter (1996) Sorcellerie et politique : les pièges du rapport élitesvillage, Politique africaine, 63, pp. 82-96.

Godin Marie (2011) Dynamiques et profils de la diaspora nigérienne en Belgique, rapport MIDA pour I'Organisation Internationale des Migrations, $58 \mathrm{p}$.

Grégoire Emmanuel (1986) Les Alhazai de Maradi. Histoire d'un groupe de riches marchands sahéliens, Paris, Éditions de I'ORSTOM, 228 p.

Grégoire Emmanuel et Labazée Pascal (1993) Grands commerçants d'Afrique de l'Ouest. Logiques et pratiques d'un groupe d'hommes d'affaires contemporains, Paris, Karthala et ORSTOM, 264 p.

Halbwachs Maurice (1941) La Topographie légendaire des Évangiles en Terre sainte. Étude de mémoire collective, Paris, Presses Universitaires de France, 206 p.

Howorth Carole, Tempest Sue and Coupland Christine (2005) Rethinking entrepreneurship methodology and definitions of the entrepreneur, Journal of Small Business and Enterprise Development, 12 (1), pp. 24-40.

Igué John O. et Soulé Bio G. (1992) L'État-entrepôt au Bénin. Commerce informel ou solution à la crise?, Paris, Karthala, $210 \mathrm{p}$.

Jarillo Carlos and Stevenson Howard (1990) A Paradigm of Entrepreneurship: Entrepreneurial Management, Strategic Management Journal, 11, pp. 17-27.

Kivisto Peter and Faist Thomas (2010) Beyond a Border. The Causes and Consequences of Contemporary Immigration, London, Sage, 310 p.

Kloosterman Robert, Van der Leun Joanne and Rath Jan (1999) Mixed embeddedness: (In)formal Economic Activities and Immigrant Business in the Netherlands, International Journal of Urban and Regional Research, 23, pp. 252-266. 
Kopytoff Igor (1986) The cultural biography of things: commoditization as process, in Arjun Appadurai Ed., The social life of things. Commodities in cultural perspective, Cambridge, Cambridge University Press, pp. 64-94.

Light Ivan (2007) Global entrepreneurship and transnationalism, in Leo-Paul Dana Ed., Handbook of research on ethnic minority entrepreneurship: a co-evolutionary view on resource management, Cheltenham, Edward Elgar Publishing, pp. 3-15.

Light Ivan (2005) The Ethnic Economy, in Neil J. Smelser and Richard Swedberg Eds., The Handbook of Economic Sociology, Princeton, Princeton University Press, pp. 650-677.

Light Ivan (1972) Ethnic Enterprise in America: Business and Welfare among Chinese, Japanese and Blacks, Los Angeles, University of California Press, 209 p.

Light Ivan and Karageorgis Stavros (1994)The Ethnic Economy, in Neil J. Smelser and Richard Swedberg Eds., The Handbook of Economic Sociology, Princeton, Princeton University Press, pp. 647-671.

Mac Gaffey Janet (1987) Entrepreneurs and Parasites. The Struggle for Indigenous Capitalism in Zaïre, Cambridge, Cambridge University Press, 241 p.

Ma Mung Emmanuel (1994) L'entreprenariat ethnique en France, Sociologie du travail, 36 (2), pp. 185-209.

Ma Mung Emmanuel (1996) Entreprise économique et appartenance ethnique, Revue Européenne des Migrations Internationales, 12 (2), pp. 211-231.

Manry Véronique (2005) Les mobilités féminines maghrébines dans l'espace euro-méditerranéen. Quand Fatima, Assia, Meryem et les autres prennent la route..., Migrations société, 17 (99-100), pp. 201-213.

Martiniello Marco, Rea Andrea, Timmerman Christiane et Wets Johan (Éds.) (2010) Nouvelles migrations et nouveaux migrants en Belgique, Gent, Academia Press, $340 \mathrm{p}$.

McCabe Ina Baghdiantz, Harlaftis Gelina et Minoglou loanna Pepelasis (Éds.) (2005) Diaspora Entrepreneurial Networks. Four Centuries of History, Oxford, Berg, $440 \mathrm{p}$.

Missaoui Lamia (1995) Généralisation du commerce transfrontalier : petit ici, notable là-bas, Revue Européenne des Migrations Internationales, 11 (1), pp. 53-75.

Peraldi Michel (2007) Aventuriers du nouveau capitalisme marchand. Essai d'anthropologie de I'éthique mercantile, in Fariba Adelkhah et Jean-François Bayart Éds., Voyages du développement. Émigration, commerce, exil, Paris, Karthala, pp. 73-113.

Polanyi Karl (1983 [1944]) La grande transformation. Aux origines politiques et économiques de notre temps, Paris, Gallimard, 467 p.

Portes Alejandro, Guarnizo Luis E. and Haller William (2002) Transnational Entrepreneurs: An Alternative Form of Immigrant Economic Adaptation, American Sociological Review, 67 (2), pp. 278-298.

Portes Alejandro and Manning Robert D. (1986) The Immigrant Enclave: Theory and Empirical Examples, in Susan Olzak and Joane Nagel Eds., Competitive Ethnic Relations, Orlando, Feborida Academic Press, pp. 47-68. 
Portes Alejandro and Zhou Min (1992) Gaining the Upper Hand: Economic Mobility among Immigrant and Domestic Minorities, Ethnic and Racial Studies, 15 (4), pp. 491-522.

Ramachandran Vijaya and Shah Manju Kedia (1999) Minority Entrepreneurs and Firm Performance in Sub-Saharan Africa, The Journal of Development Studies, 36 (2), pp. 71-87.

Riccio Bruno (2001) From "ethnic group" to "transnational community"? Senegalese migrants' ambivalent experience and multiple trajectories, Journal of Ethnic and Migration Studies, 27 (4), pp. 583-599.

Rosenfeld Martin (2012) Mobility and social capital among Lebanese and Beninese entrepreneurs engaged in transnational trade, International Review of Sociology, 22 (2), pp. 211-228.

Rosenfeld Martin (2009) Le commerce d'exportation de véhicules d'occasion entre Bruxelles et Cotonou, Les cahiers de I'Urmis, 12, [en ligne]. URL : http:// urmis.revues.org/index838.html

Schmoll Camille (2012) The making of a transnational marketplace. Naples and the impact of Mediterranean cross-border trade on regional economies, Cambridge Journal of Regions Economy and Society, 5 (2), pp. 221-238.

Schumpeter Joseph (1935 [1912]) Théories de l'évolution économique. Recherches sur le profit, le crédit, l'intérêt et le cycle de la conjoncture, Paris, Dalloz, 535 p.

Simmel Georg (2004 [1908]) Digressions sur l'étranger, in Yves Grafmeyer et Isaac Joseph Eds., L'école de Chicago : naissance de l'écologie urbaine, Paris, Flammarion, pp. 53-59.

Streiff-Fénart Jocelyne (1997) Les recherches interethniques en France. Le renouveau ?, Migrants-Formation, 109, pp. 48-65.

Swedberg Richard (2010)The Social ScienceView of Entrepreneurship: Introduction and Practical Applications, in Richard Swedberg Ed., Entrepreneurship. The Social Science View, Oxford, Oxford University Press, pp. 7-44.

Tarrius Alain (2001) Au-delà des États-nations : des sociétés de migrants. Revue Européenne des Migrations Internationales, 17 (2), pp. 37-61.

Tarrius Alain (1993) Territoires circulatoires et espaces urbains, in Mirjana Morokvasic et Hedwig Rudolph Éds., Migrants. Les nouvelles mobilités en Europe, Paris, L'Harmattan, pp. 93-117.

Tarrius Alain (1992) Les fourmis d'Europe. Migrants riches, migrants pauvres et nouvelles villes internationales, Paris, L'Harmattan, 208 p.

Waldinger Roger, Aldrich Howard and Ward Robin (Eds.) (2006) Ethnic Entrepreneurs. Immigrant Business in Industrial Societies, London, Sage, 234 p.

Warnier Jean-Pierre (1993) L'esprit d'entreprise au Cameroun, Paris, Karthala, $312 \mathrm{p}$.

Zhou Min (2007) Revisiting Ethnic Entrepreneurship: Covergencies, Controversies, and Conceptual Advancements, in Alejandro Portes and Josh DeWind Eds., Rethinking Migration. New Theoretical and Empirical Perspectives, New York, Berghahn Books, pp. 219-253. 


\section{Martin Rosenfeld}

\section{$\cdots$ Entrepreneurs transnationaux et commerce d'exportation de véhicules d'occasion : la filière Bruxelles-Cotonou}

Cet article s'intéresse au profil migratoire des importateurs de véhicules d'occasion de la filière Bruxelles-Cotonou. Ces deux villes occupent une position de places marchandes au sein d'une filière commerciale transnationale. Après une rapide revue de la littérature, le concept d'entrepreneur migrant est mobilisé pour décrire les importateurs de véhicules. Les nombreuses différences observées entre les importateurs libanais et béninois permettent une analyse comparative. Une discussion théorique est engagée sur les concepts les plus à même de décrire le profil migratoire de ces entrepreneurs. Il apparaît ainsi que les importateurs libanais ont un profil de diaspora commerciale mettant à profit une appartenance ethnique commune pour développer leur activité économique. Les importateurs béninois, quant à eux, s'apparentent davantage à des entrepreneurs transfrontaliers actifs au sein d'un territoire circulatoire.

\section{Transnational Entrepreneurs and Second-hand Cars Business between Brussels and Cotonou}

This contribution focuses on the migratory profiles of second-hand cars importers operating between Brussels, Belgium and Cotonou, Benin. Those two cities are business hubs in a transnational import-export business. After a quick state of the art, the concept of migrant entrepreneur is used to describe those second-hand cars importers. Numerous differences between Lebanese and Beninese importers allow us to draft a comparative analysis between their migratory profiles. On the one hand, Lebanese importers have a status of trade diaspora. They use their common ethnic belonging to address technical challenges linked to a common commercial activity. On the other hand, native Beninese car importers have the status of cross-border entrepreneurs working in a territory of movement between various business hubs.

\section{$\cdots$ Empresarios transnacionales y comercio de exportación de vehículos usados: la sucursal Bruselas-Cotonou}

Esta contribución se interesa en el perfil migratorio de importadores de vehículos usados de la sucursal en Bruselas, en Cotonou. Ambas ciudades ocupan una posición en el sector comercial a nivel transnacional. Después de una breve revisión de la literatura, el concepto de empresario migrante será empleado para describir a los importadores de vehículos. Las diversas diferencias observadas entre los importadores libaneses y benineses permiten un análisis comparativo. Una discusión teórica será emprendida sobre los conceptos clave a fin de describir el perfil migratorio de estos empresarios. De esta forma, pareciera que los importadores libaneses tienen un perfil de diáspora comercial aprovechando una etnicidad común para desarrollar su actividad económica, mientras que, por su lado, los importadores benineses parecen más empresarios transfronterizos activos al interior de un territorio circulatorio. 